\title{
Entangling many-body bound states with propagative modes in Bose-Hubbard systems
}

\author{
Mario Collura, ${ }^{1}$ Helge Aufderheide, ${ }^{2}$ Guillaume Roux, ${ }^{3,}$, and Dragi Karevski ${ }^{1, \dagger}$ \\ ${ }^{1}$ Institut Jean Lamour, dpt. P2M, Groupe de Physique Statistique, \\ Nancy-Université CNRS, B.P. 70239, F-54506 Vandoeuvre les Nancy Cedex, France \\ ${ }^{2}$ Department Biological Physics, Max-Planck-Institute for the Physics of Complex Systems, Noethnitzer Strasse 38, 01187 Dresden, Germany \\ ${ }^{3}$ Laboratoire de Physique Théorique et Modèles statistiques, \\ Université Paris-Sud, CNRS, UMR8626, 91405 Orsay, France
}

\begin{abstract}
The quantum evolution of a cloud of bosons initially localized on part of a one dimensional optical lattice and suddenly subjected to a linear ramp is studied, realizing a quantum analog of the "Galileo ramp" experiment. The main remarkable effects of this realistic setup are revealed using analytical and numerical methods. Only part of the particles are ejected for a high enough ramp, while the others remain self-trapped. Then, the trapped density profile displays rich dynamics with Josephson-like oscillations around a plateau. This setup, by coupling bound states to propagative modes, creates two diverging condensates for which the entanglement is computed and related to the equilibrium one. Further, we address the role of integrability on the entanglement and on the damping and thermalization of simple observables.
\end{abstract}

PACS numbers: 67.85.-d,03.75.Gg,03.75.Lm,67.85.Hj

The last decade breakthrough experiments on ultra-cold atomic gases have revived the field of strongly correlated many-body quantum systems [1], especially within nonequilibrium aspects [2]. The very low dissipation rate and long time phase coherence of these systems allow the investigation of genuine quantum effects, the role of integrability on thermalization properties, and the possibility to engineer desired states. Two examples are particularly relevant for this study. The first is Bloch oscillations (BO) [3] which occur when a particle travels on a lattice and experiences a constant external force $F$ (potential ramp): Its momentum drifts with time according to $q(t)=q(0)+F t$ (we set $\hbar$ and the lattice spacing equal to one), but modulo the Brillouin zone, setting the BO period $\tau_{B}=2 \pi /|F|$. They have been observed in many physical domains : semiconductors [4], thermal gases [5], photonics [6], cold atoms [7], and phonons [8]. BO can survive to the many-body regime, with a damping possibly related to the integrability of the model [9, 10]. The second example is the release of atoms from a trap, a standard protocol with cold atoms. Yet, keeping the optical lattice on during the expansion allows one to handle metastable states [11], or study transport phenomena in disordered potentials [12]. We lastly stress that ultra-cold atoms, beyond their fundamental interest, could be used as devices to mimic optics experiments [13] or semi-conductor physics [14].

In this paper, we put forward an experimentally realistic setup which ejects interacting bosons living on an optical lattice using a linear potential (see Fig. 1). This "Galileo ramp" experiment displays remarkable features that are understood with analytical and numerical calculations. Only part of the particles is ejected and forms a wave-packet which shape and number of particles are determined. Thanks to the initial correlations, these traveling particles remain strongly entangled with the ones remaining self-trapped in the initial

guillaume.roux@u-psud.fr

† dragi.karevski@ijl.nancy-universite.fr region, hence creating two diverging and entangled manybody condensates. In the self-trapping region, particles exhibit Josephson-like oscillations reminiscent of BO, with a density plateau due to many-body interferences, and damping controlled by the integrability of the model. The setup is particularly versatile, in comparison to analog proposals to create wave-packets [15], and relies on the general idea of coupling bound states to the propagative modes of a lattice. Results in the hard-core bosons limit provides a quantitative and intuitive understanding which allows for straightforward generalizations (mirrors, beam-splitters,...).

\section{SETUP DESCRIPTION}

\section{A. Hamiltonian}

We describe the setup by means of the one-dimensional (1D) Bose-Hubbard model (BHM)

$\mathcal{H}=-J \sum_{j}\left[b_{j+1}^{\dagger} b_{j}+\right.$ h.c. $]+\frac{U}{2} \sum_{j} n_{j}\left(n_{j}-1\right)+\sum_{j} V_{j}(t) n_{j}$

where $b_{j}^{\dagger}$ is the bosonic creation operator at site $j, n_{j}=b_{j}^{\dagger} b_{j}$ is the density operator. $J$ and $U$ are respectively the hopping and interaction magnitudes while $V_{j}(t)$ is the time-dependent external potential. At times $t<0$, the potential is a deep box of width $A$ which confines all $N$ particles in region $\mathcal{A}$, with

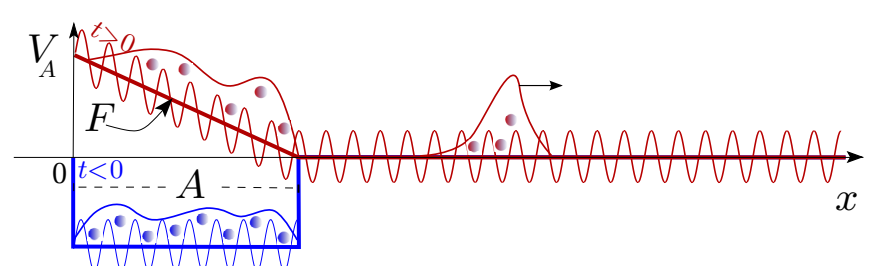

FIG. 1. (color online) Sketch and notations of the considered setup. 
the average density $\rho=N / A$. At times $t \geq 0$ (quench), the potential is suddenly changed to a linear ramp $V_{j}=V_{A}-F j$ in region $\mathcal{A}$ and zero elsewhere (see Fig. 11). We provide in Appendix A the criteria to neglect tunneling toward the upper Bloch band.

For simulations, the setup is embedded in a larger box of size $L$, and the gas is assumed to be isolated. Its dynamics is studied via analytical methods in the non-interacting and Hard-Core Boson (HCB) $U=\infty$ limits. HCB dynamics can be solved exactly using the mapping to a fermion Hamiltonian and diagonalizing the single-particle physics (see below). Observables are then expressed as determinants that are computed numerically. The out-of-equilibrium calculations are based on Refs. 16 and 17. Otherwise, two ab-initio techniques are used to compute observables: Lanczos diagonalization and time-dependent density-matrix renormalization group (tDMRG) [18-21] (see also Refs. 22 for a good introduction), which is particularly useful to compute the entanglement entropy of Fig. 4. Lanczos diagonalization are used with a cutoff $M$ in the onsite boson number. $M=N$ is taken on the box configuration (except for $L=18$ for which $M=7$ ). For $L=32$, the 64bits limitation imposes $M=3<N$. This can participate in the little difference between the free bosons analytics and the numerics in Fig. 2(d). tDMRG is performed with $M=2$. After sweeping through the chain to apply the evolution operator, we keep the maximal value of the block entanglement entropy $S_{\max }(t)$ and we update the number of kept states $m$ at time $t+d t$ to $m(t+d t)=\operatorname{int}\left[m(t) \times \exp \Delta S_{\max }(t)\right]$, in order to account for the eventual growth of entanglement. Typically, starting the evolution with $m(0) \in[16,24]$, we finish with $m(T) \in[24,110]$ depending on the value of $U$ and $F$. Comparisons with Lanczos calculations ensured that this is sufficient to monitor errors for the considered parameters. For these ab-initio methods, the Hilbert sizes at play are not challenging and the numerical errors are under control.

\section{B. Hydrodynamic description of HCB}

In the limit $N, A \gg 1$, after a Jordan-Wigner transformation, a chain of HCB maps to the free fermion Hamiltonian $\mathcal{H}=\sum_{q} \varepsilon_{q} \eta_{q}^{\dagger} \eta_{q}$ with the fermionic operators $\eta_{q}$ of momentum $q \in[-\pi, \pi]$. The kinetic part of the energy, $-\Delta \cos q$ with $\Delta=2 J$, builds up a $1 \mathrm{D}$ band of width $2 \Delta$ and gives a velocity $v(q)=\Delta \sin q$. The ground-state is the corresponding Fermi sea of Fermi momentum $q_{F}=\pi \rho$. This is a good description of the initial state provided the well is large and deep enough. The features of the density dynamics are well captured by the hydrodynamic limit using the continuous position variable $x$. The initial state has approximately a coarse grained phasespace density $w(x, q)=\frac{1}{2 \pi} \Pi_{[0, A]}(x) \Pi_{\left[-q_{F}, q_{F}\right]}(q)$, where $\Pi_{[a, b]}$ is the characteristic function over the interval $[a, b]$. After the sudden quench, the energy of a particle at position $x$ is shifted by the additional potential value $V(x)$. The dynamics being unitary, quasi-particles are emitted to the right and to the left on a trajectory of constant energy $\varepsilon$. The density of particles $\rho(x)$ is the sum of the densities of these right and left movers, to which are attributed the local velocities $v( \pm q(x))$, where $q(x)=\arccos [(V(x)-\varepsilon) / \Delta]$. This approach allows us to reconstruct the evolving density profile $\rho(x ; t)$.

\section{WAVE-PACKET EMISSION OF THE GALILEO RAMP}

\section{A. Wave-packet density profile}

The typical evolution of the density of HCB after the quench for increasing forces is given on Fig. 2.a). Only part of the bosons are ejected, with maximum velocity $\Delta$, and the wave-packet spreads with time. In the HCB hydrodynamic description, the population with energy $\varepsilon \in[-\Delta, \Delta]$ is connected to the propagating states outside $\mathcal{A}$ (see Fig. 2(b)). These particles escape from region $\mathcal{A}$ and propagate toward $\infty$. As for BO, the momentum of a right(left) mover of energy $\varepsilon$ obeys $q^{ \pm}(t)= \pm q+F t$. From energy conservation, it converts all its potential energy into kinetic energy when it reaches the position $A$ at a time $t^{ \pm}$, given by $F t^{ \pm}=\mp q+\arccos (-\varepsilon / \Delta)$. After escaping from $\mathcal{A}$, both travels at constant velocity, giving the ballistic trajectory $x^{ \pm}(t ; q, \varepsilon)=\sqrt{\Delta^{2}-\varepsilon^{2}}\left(t-t^{ \pm}\right)+A$. At times larger than $\tau_{B}$, these particles have all left region $\mathcal{A}$ and their density profile is the sum of the right and left movers contributions

$$
\rho_{\mathrm{esc}}^{ \pm}(x ; t)=\int_{-\Delta}^{\Delta} \frac{d \varepsilon}{F} \int_{Q(\varepsilon)} \frac{d q}{2 \pi} \delta\left(x-x^{ \pm}(t ; q, \varepsilon)\right),
$$

in which $Q(\varepsilon)$ refers to the yellow domain of Fig. 2(b). Fig. 2(b) shows the very good agreement, up to small quantum interference effects, between exact diagonalization data and the hydrodynamic prediction (1).

\section{B. Number of ejected particles}

The total number $N_{\mathrm{esc}}^{\mathrm{HCB}}$ of escaping $\mathrm{HCB}$ is readily obtained by integrating the initial density on the same domain

$$
\rho(x \leq A, \varepsilon ; t=0)=\int_{Q(\varepsilon)} \frac{d q}{\pi} \delta(\varepsilon-V(x)+\Delta \cos q) .
$$

One obtains that, for a small positive force $F \leq \Delta(1+$ $\left.\cos q_{F}\right) / A$, all particles are connected with propagative states, i.e. $N_{\text {esc }}^{\mathrm{HCB}}=N$. When increasing $F$ beyond, some particles remain trapped in region $\mathcal{A}$ and the explicit calculation yields:

$$
\left.N_{\mathrm{esc}}^{\mathrm{HCB}}=\frac{\Delta}{\pi F}\left[q_{F}+\sin q_{F}\right)-g(F A / \Delta)\right]
$$

with $g(x)=\sqrt{x(2-x)}-(x-1) \arccos (x-1)$ when $\Delta\left(1+\cos q_{F}\right) / A \leq F \leq 2 \Delta / A$, and $g(x)=0$ when $F \geq 2 \Delta / A$. A comparison with simulations is displayed on Fig. 2.c), demonstrating the accuracy of the approach.

Lowering interactions naturally modifies $N_{\text {esc }}$. Taking the opposite case of free bosons, this single-particle physics is solved along the same lines : free bosons are initially gathered 

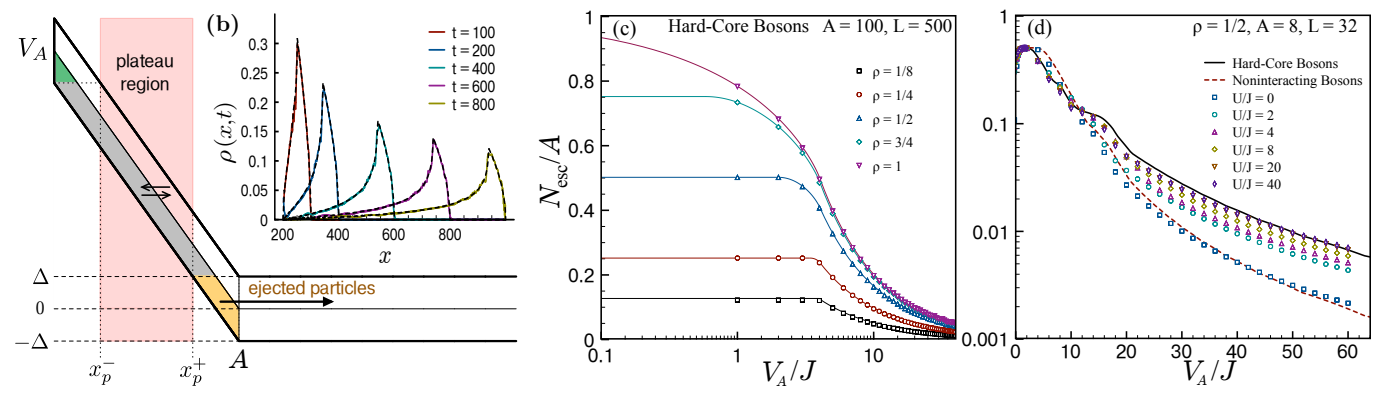

FIG. 2. (color online) (a) time-evolution of the density profile at half-filling for increasing ramp height $V_{A}=1,3,5,8[J]$. (b) sketch explaining the hydrodynamic calculations. Inset: numerical escaping profiles (full lines) compared to the hydrodynamic approach (dashed lines). (c) number of escaping HCB particles vs. $V_{A}$ for various initial densities $\rho$ (hydrodynamic predictions in full lines). (d) same number, varying the interaction strengths $U / J$ at half-filling, and compared to analytical limiting cases.

in the ground-state wave-function of the deep well so that the initial density profile in region $0 \leq x \leq A$ is

$$
\rho(x ; 0)=N \frac{2}{A} \sin ^{2}\left(\frac{\pi x}{A}\right) .
$$

In this regime, all bosons share the same dynamics and the density profiles of the trapped and outgoing wave-packets depend only trivially on $N$ which enters as a prefactor. The initial state thus consists of $N$ bosons of initial momentum $q_{0} \simeq \pi / A$. The particles leaving the confinement region are again those with energy $\varepsilon \in[-\Delta, \Delta]$, corresponding to the space interval $\vartheta=[\max (0, A-2 \Delta / F), A]$. Then, one obtains $N_{\text {esc }}^{\text {free }}=N$ for $0 \leq F \leq 2 \Delta / A$ and

$$
N_{\mathrm{esc}}^{\mathrm{free}}=N\left[\frac{2 \Delta}{F A}-\frac{1}{2 \pi} \sin \left(2 \pi \frac{2 \Delta}{F A}\right)\right] .
$$

for $F \geq 2 \Delta / A$. This time, the strong force scaling is $F^{-3}$. This last result is also valid for $N=1$, in which case the single-particle wave-function is in a superposition of bound and diffusive states. In Fig. 2(d), numerical simulations for various $U$ shows how results interpolate between these two limiting cases with an interaction-dependent behavior at large $F$. Note that, surprinsingly, interactions do not favor escaping at small forces.

\section{DYNAMICS OF TRAPPED PARTICLES}

\section{A. Density profile}

As seen from Fig. 2(a), the trapped HCB exhibit a rather rich physics with oscillations reminiscent of $\mathrm{BO}$. This can be understood in the hydrodynamic approach: We consider the main contribution coming from trapped particles with energies $\varepsilon \in\left[\Delta, V_{A}-\Delta\right]$ (grey area in Fig. 2(b)). Neglecting tunneling escapes at the band edges, the corresponding densities of right/left movers are

$$
\rho_{\mathrm{coh}}^{ \pm}(x ; t)=\int_{0}^{q_{F}} \frac{d q}{2 \pi} \int_{\Delta}^{V_{A}-\Delta} d \varepsilon \delta(\varepsilon-V(x)+\Delta \cos (q \pm F t))
$$

These are clearly periodic functions of time, oscillating with period $\tau_{B}$. By introducing $\tilde{q}=q \pm F t$ in each term and integrating over energies, the coherent part of the density reads

$$
\rho_{\mathrm{coh}}(x ; t)=\int_{F t-q_{F}}^{F t+q_{F}} \frac{d \tilde{q}}{2 \pi} \Pi_{\left[\Delta, V_{A}-\Delta\right]}(V(x)-\Delta \cos \tilde{q}) .
$$

From (5), we see that oscillations are present provided $\rho<1$ (superfluid regime of HCB). Indeed, at unit filling $\rho=1$ (Mott state) $q_{F}=\pi$ and the integration range then covers a full period, leading to a static trapped density profile. The density profile can actually be computed at any time $t$ from (5) (see below), and a comparison with numerics is given in Fig. 3. a). We first observe that incoherent contributions due to high energy modes of energy $\varepsilon \in\left[V_{A}-\Delta, V_{A}+\Delta\right]$ (green area in Fig. 2(b)) not taken into account in (5) increase both the average and the fluctuations of the particle density on the left side. Indeed, due to the free boundary at $x=0$, high energy particles sharing initially the same momentum $q$ are not reflected at $x=0$ at the same time. Consequently, a dephasing appears between them and their contribution to the total spatial density is somehow incoherent. A simple way to suppress this incoherent effect is to eject these particles into a propagative band on the left $(x<0)$, as shown in Fig. 3 b) where we see a very good agreement, up to small interference effects, between the exact numerical results and the hydrodynamic predictions when the incoherent particles are removed.

Another striking feature of Fig. 3. (a)-(b) is the existence of a stationary density plateau: The argument of the $\Pi$ function in (5) reaches its edges for positions $x_{\text {inf/sup }}$ given by $V\left(x_{\text {inf }}\right)-\Delta \cos \tilde{q}=V_{A}-\Delta$ and $V\left(x_{\text {sup }}\right)-\Delta \cos \tilde{q}=\Delta$. For max $x_{\text {inf }} \leq x \leq \min x_{\text {sup }}$, the integral is independent of time and the initial density plateau survives. Therefore, the condition to have this plateau is $F>4 \Delta / A$ and its width is $A-4 \Delta / F$. The boundaries of the plateau are given by $x_{p}^{-}=\max x_{\text {inf }}$ and $x_{p}^{+}=\min x_{\text {sup }}$, which can be written

$$
x_{p}^{ \pm}=\frac{A}{2} \pm\left(\frac{A}{2}-\frac{2 \Delta}{F}\right) .
$$

At each sides of the plateau, an excess density oscillates, being at the left(right) for (half-)integer multiples of $\tau_{B}$. 

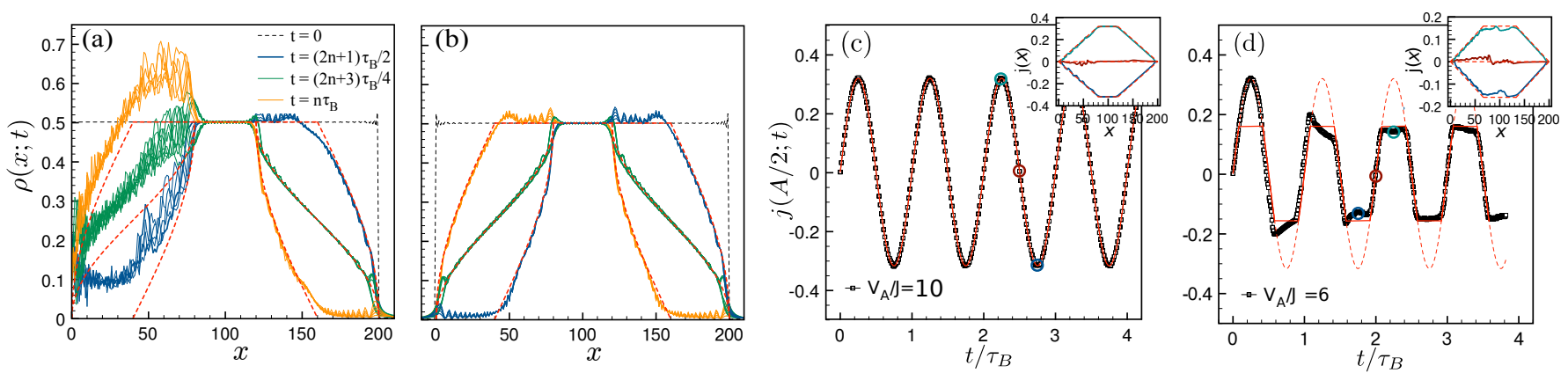

FIG. 3. (color online) Density profile without (a) and with (b) a propagating band opened at the left of $x=0$ at different times for $V_{A} / J=10$, $A=200$ at half-filling $\rho=1 / 2$ (hydrodynamic predictions are given by the dashed red lines). (c) Corresponding current density at $x=A / 2$. Inset: Current profiles at different times (colors refer to the circles). Full lines are the exact results while dashed red lines are the hydrodynamic predictions. (d) same but for a smaller ramp $V_{A} / J=6$. The straight red line is the expected truncated sine function while the dashed red one is the pure sine oscillation (see the main text).

The explicit density profile is easily computed at any time $t$ from Eq. 5]. For example, at integer multiples of the BO period $\tau_{B}$, the distribution of the trapped bosons is shifted maximally to the left (down to $x_{\text {inf }}\left(q_{F}\right)<x_{p}^{-}$) and is given by

$$
\rho_{\mathrm{coh}}\left(x ; n \tau_{B}\right)= \begin{cases}\frac{1}{\pi} \arccos \left[\left(V(x)-V_{A}\right) / \Delta+1\right] & 0 \leq x \leq x_{\mathrm{inf}}\left(q_{F}\right) \\ \rho & x_{\mathrm{inf}}\left(q_{F}\right) \leq x \leq x_{p}^{+} \\ \rho-\frac{1}{\pi} \arccos [V(x) / \Delta-1] & x_{p}^{+} \leq x \leq x_{\mathrm{sup}}\left(q_{F}\right) \\ 0 & x_{\mathrm{sup}}\left(q_{F}\right) \leq x \leq A\end{cases}
$$

The distribution then propagates from this macroscopic left state to the macroscopic right one (reached at half periods times $t=(n+1 / 2) \tau_{B}$ with integer $\left.n\right)$. The spatial profile of the right state is simply deduced from the left one by the transformation $x \rightarrow A-x$, using the mirror symmetry at $A / 2$. These are the results plotted in Fig. 3. $\mathrm{a}-\mathrm{b})$.

\section{B. Collective Josephson-like oscillations}

This remarkable pendulum motion of the density is naturally associated with a flow of particles through the plateau region. The flow of particles from the left to the right of the trapping zone and vice versa gives rise to a periodic current density $j(x ; t)$ which, in the hydrodynamic limit, is simply the sum over all momenta of $\rho(q) v(q)$, i.e. the quasi-particle currents $\left[\rho^{+}(q)-\rho^{-}(q)\right] \Delta \sin q$. In the trapped region the current density is given by the integral expression

$$
j(x ; t)=\Delta \int_{F t-q_{F}}^{F t+q_{F}} \frac{d \tilde{q}}{2 \pi} \sin \tilde{q} \Pi_{\left[\Delta, V_{A}-\Delta\right]}(V(x)-\Delta \cos \tilde{q}) .
$$

For $F>4 \Delta / A$, in the plateau region $\Omega$ one has simply from 7) a spatially constant current (which was expected from the continuity equation since the local density is constant in time)

$$
j(x ; t)=\frac{\Delta}{\pi} \sin \left(q_{F}\right) \sin (F t),
$$

which oscillates harmonically with period $\tau_{B}$. The maximum current amplitude is obtained at half filling when $q_{F}=\pi / 2$, while the current is exactly zero in the Mott phase (unitary filling giving $q_{F}=\pi$ ) reflecting the stationarity in time of the trapped density in that case. These Josephson-like oscillations are reported in Fig. 3 (c) and we see that the hydrodynamic description matches perfectly the numerical data. Outside the plateau, the current density has a spatial variation which is simply deduced from its integral representation (7). Again, as seen in the inset of Fig. 3 (c), the hydrodynamic description is very good.

At lower forces, for $2 \Delta / A<F<4 \Delta / A$, when there is no more a plateau region but still self-trapped particles, the temporal evolution of the current is no more given by a pure sine function due to the interplay between the integration interval and the support of the door function entering into (7). For example, at half filling $q_{F}=\pi / 2$ and taking $V_{A}=3 \Delta$, one has from (7) in the middle of the ramp

$$
j\left(\frac{A}{2}, t\right)=\left\{\begin{array}{ll}
\frac{\Delta}{\pi} \sin (F t) & \text { for }|\sin (F t)| \leq\left(V_{A} / 2 \Delta-1\right) \\
\Delta / 2 \pi & \text { for }|\sin (F t)| \geq\left(V_{A} / 2 \Delta-1\right)
\end{array} .\right.
$$

This truncated sine essentially reflects the escape of particles which were initially located in the middle of the condensate. Indeed, for $V_{A}<4 \Delta$ the escape locus $x_{p}^{+}$extends over the middle of the ramp, since $x_{p}^{+}<A / 2$, leading to a lowering of the current intensity after the corresponding particles have been ejected. As a support of this, notice on Fig. 3(d) how dur- 
ing the first half period, as the particles are moving from the left to the right and have not yet abandoned the condensate, the current shows a perfect sinusoidal signal which gets truncated as time goes on. Notice that, despite a behavior similar to the Josephson effect, the oscillations stem from a fundamentally different effect - many-body interferences with strong interactions - and without a tunneling barrier - the plateau develops within the system.

\section{ENTANGLEMENT ENTROPY BETWEEN THE WAVE-PACKETS}

After the quench, the condensate is split into two entangled pieces moving apart: the escaping particles and the self-trapped ones. This entanglement between bound states and propagative ones can be quantified through the bipartite von-Neuman entropy $S_{t}(A)=-\operatorname{Tr}\left\{\varrho_{t}(A) \ln \varrho_{t}(A)\right\}$, where $\varrho_{t}(A) \equiv \operatorname{Tr}_{>A}\{|\Psi(t)\rangle\langle\Psi(t)|\}$. As seen on Fig. 4(a), this entropy essentially evolves up to an asymptotic value $S_{\infty}(A)$, as expected, which depends on $N, U$ and $F$. The effect of the force is qualitatively inferred from Fig. 4(a). At small $F$ when all particles are ejected (see $F=J / 4$ ), after an initial increase of the entanglement due to the crossing of position $x=A$ by the many-body wave-packet, the entanglement finally vanishes when most particles have left region $\mathcal{A}$. This small- $F$ regime is almost independent on the interaction. Increasing the force, the asymptotic entanglement first increases, passing through a maximum when approximately half of the initial density is ejected, before decreasing at larger forces $F$, simply because $N_{\text {esc }}$ falls down. The effect of interactions on $S_{\infty}(A)$ is two-fold : one is to modify $N_{\text {esc }}$ as seen on Fig. 2(d), and the other one is related to the fact that non-integrability (significant when $U \simeq J$ ) usually increases the chaoticity of excited states, leading to higher entanglement entropies. A strong enhancement of the entanglement is thus observed for $U=2 \mathrm{~J}$, by comparison to the behavior close to the integrable $\mathrm{HCB}$ limit (Fig. 4(a)). Lastly, in the inset of Fig. 4(a), we show the early evolution of $S_{t}(A)$ which is controlled by the rate at which particles are emitted. This initial emitting rate, as expected, is enhanced by the repulsion strength $U$ and by the force $F$.

The strong entanglement observed between the two pieces of the condensate must stem from the initial state in which all particles, embedded in the same condensate, are naturally entangled. Therefore, the asymptotic value $S_{\infty}(A)$ of the bipartite entropy should be related to the entanglement present initially between the particles that will leave region $\mathcal{A}$ and those that will stay. In the HCB limit, this idea actually gives a quantitative prediction for $S_{\infty}(A)$. Indeed, in Fig. 4 (b) we show $S_{t=800}(A) \simeq S_{\infty}(A)$, as a function of $V_{A}$, compared to the initial equilibrium ground-state entropy $S_{0}\left(x_{p}^{+}\left(V_{A}\right)\right)$ evaluated at the point $x_{p}^{+}\left(V_{A}\right)$ (see Fig. 2 b)) which marks the leftmost initial position of the ejected particles. The entropy $S_{0}$ is computed from exact diagonalization and also plotted from the (continuous limit) conformal field prediction [23]

$$
S_{0}(x)=\frac{1}{6} \ln \left(\frac{4 A}{\pi} \sin (\pi \rho) \sin \frac{\pi x}{A}\right)+c^{\prime},
$$

(a)

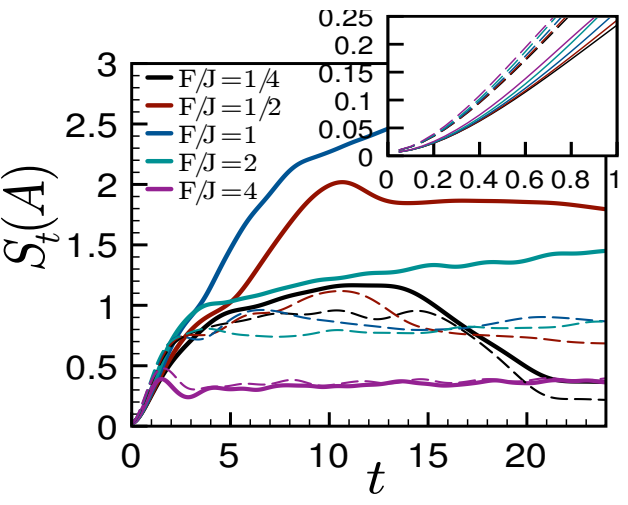

(b)

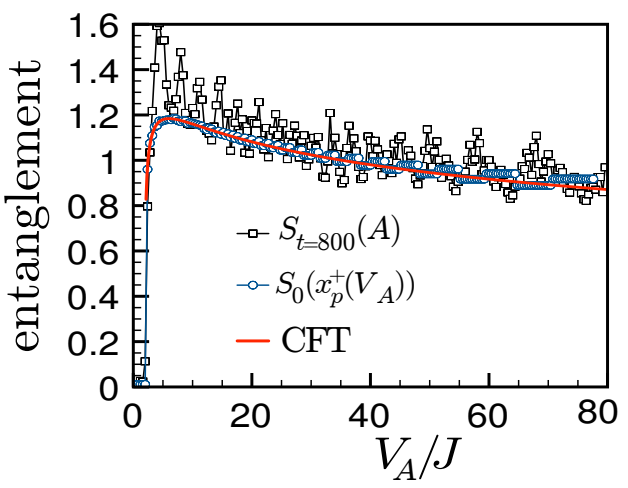

FIG. 4. (color online) (a) evolution of the entanglement entropy $S_{t}(A)$ for an increasing force $F(\rho=0.5, A=8, L=32$; full lines $U / J=2$, dashed lines $U / J=20$; Inset: short time evolution.) (b) Asymptotic value of $S_{t}(A)$ (taken at $t=800$ ) for HCB as a function of $V_{A}$ and compared with the initial bipartite entropy $S_{0}\left(x_{p}^{+}\left(V_{A}\right)\right)$ from both numerics and CFT predictions ( $\rho=0.5, A=200$ ).

with $c^{\prime} \simeq 0.25$. The good agreement found between these three curves supports the above picture. The remarkable connection between the equilibrium and non-equilibrium entanglements found in this setup could be helpful in the search for measuring many-body entanglement (9). This connection could be possibly used to infer equilibrium (local) correlations from measurements on far apart particle packets. We also stress that the setup works for few-particles physics for which the measurement of entanglement is easier.

\section{PROPOSALS ON ACHIEVING WAVE-PACKET MANIPULATIONS}

In Fig. 5, we give ideas on how to manipulate travelling wave-packets generated by the previously discussed "Galileo ramp" setup. The arguments are intuitively based on the HCB picture from energy conservation and demonstrated by numerical calculations. First, a travelling wave-packet can be catched on a lattice by suddenly increasing/decreasing the chemical potential (in a ramp shape on the figure) so that the particles cannot escape the trapping zone. It requires that the condensate is on the region before changing the potential (first sketch). If the potential is raised before the condensate arrives, then the region acts as a mirror (second sketch). If the mirror 

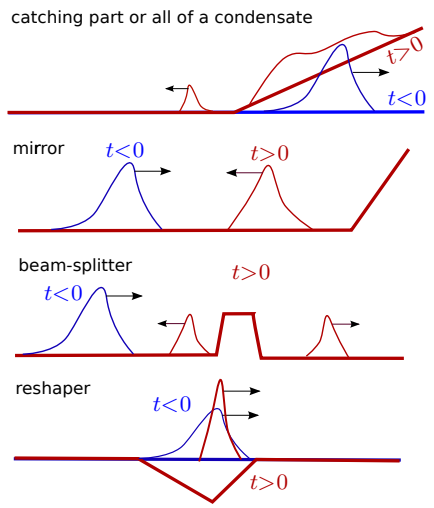
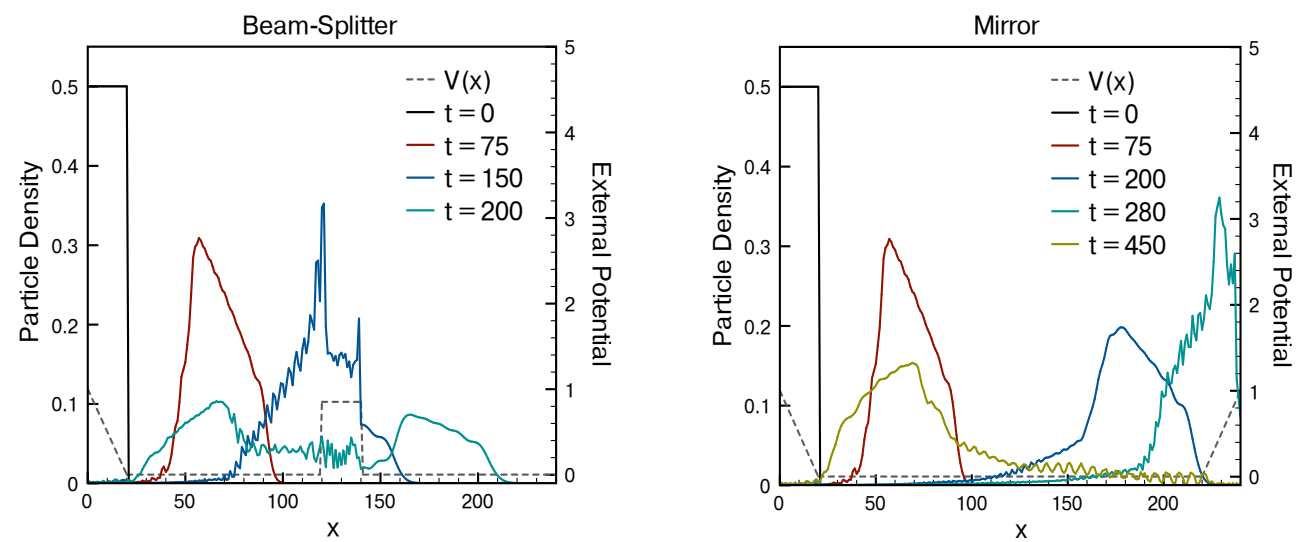

FIG. 5. (color online) Left : Proposed setups to manipulate a travelling condensate on the lattice. Middle : numerical example of the realization of a mirror (the dashed grey line represents the $t>0$ potential). Right : numerical example of the realization of a beam-splitter (same color code).

height is finite and a barrier builds up, we obtain a situation similar to single-particle tunneling process. Then, the barrier acts as a beam-splitter (third sketch). Last, applying a nonuniform potential on a wave-packet can reshape it by slowering the fastest particles and accelerating the slowest ones (fourth sketch). This would help fight the natural broadening of the wave-packet. In order to show that these intuitive behavior does take place in the real time evolution of HCB, we give in Fig. 5 two examples of numerical simulations showing the realization of a mirror and of a beam-splitter. These devices, together with the proposed source of entangled wavepackets, could help realize interferometry measurements and their applications - with the advantage that the two wavepackets can be spatially well separated, thus being able to experience different real-space paths.

\section{DAMPING AND THERMALIZATION IN THE CLOSED BOX GEOMETRY}

We see that the main physics is well captured by the integrable HCB and non-interacting limits. Still, the model is essentially non-integrable at finite $U / J$, which has some consequences on the dynamics. In order to focus on this aspect, we remove the propagative band and confine the particles in a box, thus allowing larger sizes for simulations. In this configuration, BO are damped in the chaotic regime [9] and experiments reported very low damping nearby the non-interacting point [10]. Here, we go further by computing the damping time and the distance from thermalization for all $U$. In particular, we stress that the integrable nature of the HCB limit shows up in both quantities. Therefore, we relate in this section the spectral features of the final Hamiltonian and of the quench distribution to experimentally accessible observables. In particular, we would like to see whether the (non)-integrable nature of Hamiltonian has noticeable consequences on simple observables. In addition to the damping of BO which was previously discussed in Refs. 10 and 24, we address the question of the thermalization [2].

\section{A. Time evolution after a sudden quench}

The system is prepared in the ground-state $\left|\psi_{0}\right\rangle$ of a box without the linear potential which is suddenly turned on at $t=0$. We write $E_{n}$ the energies and $|n\rangle$ the eigenvectors of the final Hamiltonian governing the dynamics. In this basis, the matrix elements of an observable $O$ are $O_{n m}=\langle m|O| n\rangle$ and the associated excitation frequencies between $n$ and $m$ are written $\omega_{n m}=E_{n}-E_{m}$. Let $c_{n}=\left\langle n \mid \psi_{0}\right\rangle$ be the coefficients of the initial state in this basis, and $p_{n}=\left|c_{n}\right|^{2}$ the corresponding weights. These weights are the quench distribution, or diagonal ensemble, and simply provide the averaged contribution of excited states to the time-evolution. The fidelity $\mathcal{F}$, defined by

$$
\mathcal{F}(t)^{2}=|A(t)|^{2} \text { with } A(t)=\left\langle\psi(t) \mid \psi_{0}\right\rangle=\sum_{n} p_{n} e^{-i \omega_{n 0} t},
$$

plays an important role in the dynamics and contains the information on the distribution through the Lehmann representation of $A(\omega)$. Given an Hermitian observable $O$, its real-time behavior and corresponding derivative read

$$
\begin{aligned}
& O(t)=\bar{O}+\sum_{n<m} 2\left|c_{n} c_{m}^{*} O_{n m}\right| \cos \left(\omega_{n m} t+\phi_{n m}\right) \\
& \dot{O}(t)=-\sum_{n<m} 2\left|c_{n} c_{m}^{*} O_{n m}\right| \omega_{n m} \sin \left(\omega_{n m} t+\phi_{n m}\right)
\end{aligned}
$$

with

$$
\bar{O}=\sum_{n} p_{n} O_{n n}
$$

the time-averaged expectation value (assuming a nondegenerate spectrum) and $\phi_{n m}$ some phases determined by the initial conditions and the observable. There are two interesting behaviors in the time-dependence : the short-time behavior, related to the damping of the observable towards $\bar{O}$, and the value $\bar{O}$ itself which can be compared to statistical ensemble predictions to check thermalization. While only the 
(a)

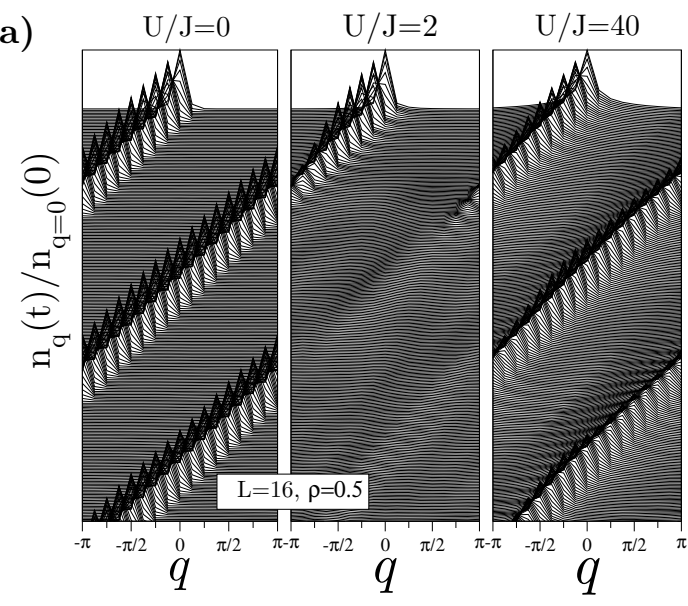

(b)

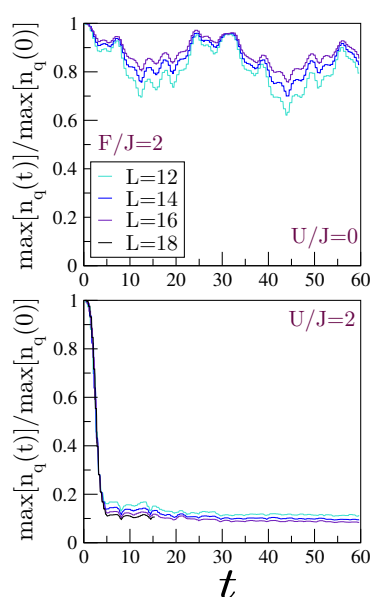

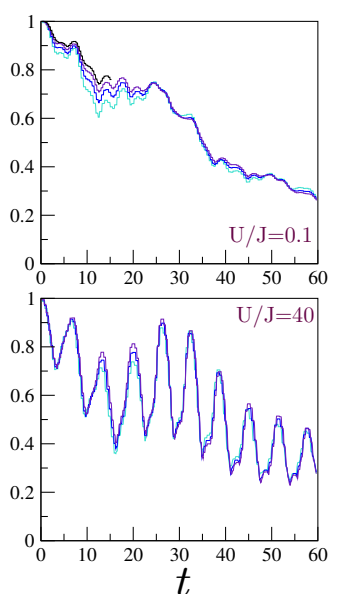

FIG. 6. (color online) (a) Evolution of the momentum distribution $n_{q}(t)$ as a function of time (vertical shift) for different $U / J$ and a fixed force $F=2 J$. The damping seems to be clearly related to the non-integrability of the model. (b) normalized damping of the momentum distribution $n_{q}(t)$ for different sizes and interactions (the signal local maximum is taken over a short time window to remove finite size oscillations associated with the $q$-space discretization).

diagonal elements enter the expression of $\bar{O}$, the off-diagonal ones $O_{n m}$ will play a role in the damping, together with the associated weights $\left|c_{n} c_{m}^{*}\right|$ and frequencies $\omega_{n m}$. These two features should depend on the integrability of the Hamiltonian through selection rules, for instance.

\section{B. Observables}

In this section, we define the observables $O$ that are used. The simplest observable is the local density $n_{j}(t)=\left\langle\hat{n}_{j}\right\rangle(t)$. Computing the one-body density-matrix $g_{j k}(t)=\left\langle b_{j}^{\dagger} b_{k}\right\rangle(t)$ allows one to access two important observables. The first one is the momentum distribution $n_{q}(t)=\left\langle\hat{n}_{q}\right\rangle(t)$, with

$$
n_{q}(t)=\frac{1}{L} \sum_{j k} e^{i q(j-k)} g_{j k}(t)
$$

which is measured using time of flight techniques. A peak in $n_{q}$ usually measures the coherence of the condensate at the corresponding wave-vector. The second one is the condensate fraction $f_{0}(t)$, defined as the largest eigenvalue of the onebody density-matrix. It matches $N$ when all bosons share the same state, and is of order $\sqrt{N}$ in the HCB regime. Even so it is not experimentally observable, the fidelity $\mathcal{F}(t)$, defined above, quantifies the distance from the initial state and is as well sensitive to the chaotic features of the Hamiltonian.

\section{Damping of observables}

The spectral features and chaoticity of the Hamiltonian are provided in Appendix $\mathrm{B}$. When one goes to the large- $U$ or large- $F$ limits, the frequencies spectrum $\omega_{n m}$ is such that they are all very close to the same level spacing ( $U$ or $F$ ), i.e. a nearly equally spaced spectrum. Of course, as judged by the poissonian nature of the spectrum at finite $J$, a dense level-spacing distribution is still present, generically leading to damping. In this regime, we expect some observables to show strong oscillatory behavior at the main frequency and its harmonics. This is a situation qualitatively related to the classical motion in closed orbits. In the quantum version, this would show up in the pseudo phase-space $(O, \dot{O}$ ) (from Eqs. (10)-(11) ) as more or less complex orbits depending on the number and weights of the harmonics involved. In the nearly equal level spacing spectrum situation, the fidelity would obviously display similar resonances (or revivals). In the case of non-integrable Hamiltonian, although level repulsion tends to rigidify the spectrum, we expect that the weights and the frequencies are spread and of the same magnitude so that the damping of observables usually occurs on shorter times. In the pseudo phase-space $(O, \dot{O})$, damping translates into a spiral structure of the orbit. We do observe this qualitative effect, particularly on the local density and its associated particle current (data not shown).

The first interesting quantity to look at is the momentum distribution v.s. time because it captures well the Bloch oscillations. We recall that, at the single-particle level, the momentum is shifted with time according to $q(t)=q(0)+F t$. In Fig. 6, we recover that $n_{q}(t)$ is globally shifted with time for all $U / J$, with a period close to the Bloch period $\tau_{B}$. Increasing $U$ further does not change much this period (hardly seeable in the numerics) and the main effect of interactions is to destroy the main peak signaling the bosonic coherence. Indeed, for $U=2 J$, we observe a strong damping of the coherence after short times. Interestingly, increasing $U$ brings us close to the HCB limit and the damping time increases again. Finally, for very large $U$, the central peak shape is preserved for very long times.

Another interesting point with the momentum distribution within this setup is that the zero-momentum evolution is qualitatively related to the fidelity of the system in the near- 


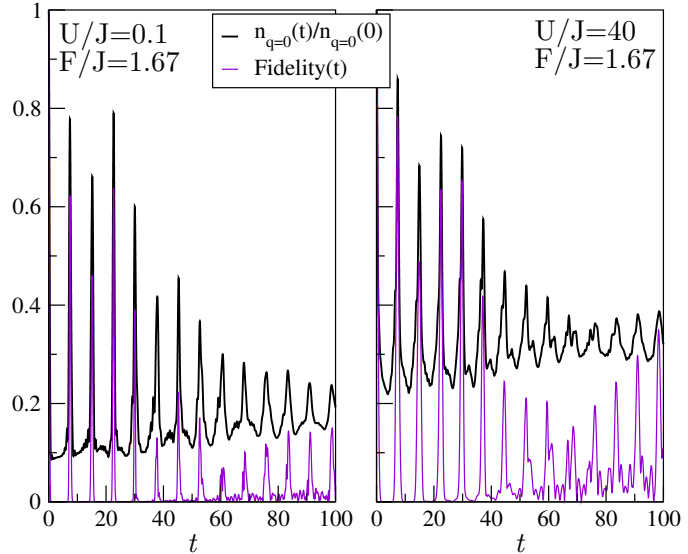

FIG. 7. (color online). Comparison between $n_{q=0}(t)$ and the fidelity in the integrable regimes, showing the revivals.

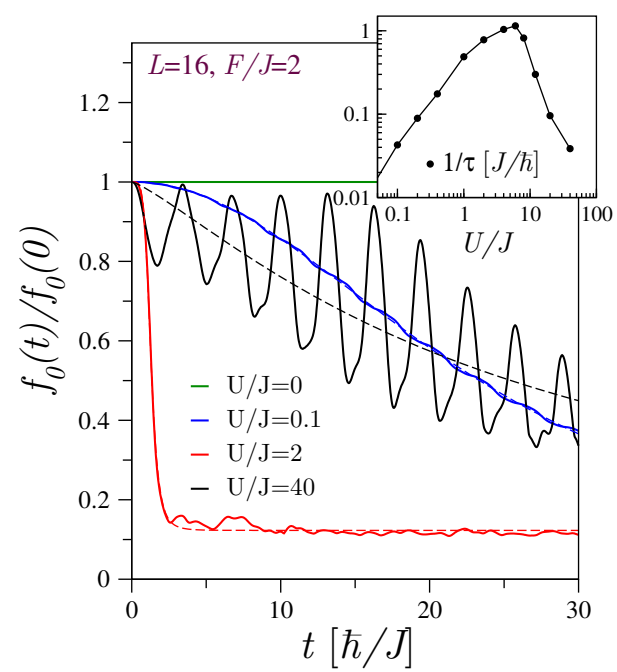

FIG. 8. (color online). Typical evolution of the condensate fraction $f_{0}(t)$ with time for various interactions and fixed $\operatorname{ramp} F=2 J$. Inset: inverse damping time $\tau$ v.s. $U / J$.

integrable regimes, both signaling strong revivals of the initial wave-function (see Fig. 77). In fact, we know that the momentum distribution gets back to its initial position in $q$-space after a period $\tau_{B}$. More than the momentum distribution, the whole many-body wave-function actually comes back close to the initial state, yielding strong revivals in the fidelity. We observe that the peaks in the fidelity and in $n_{q=0}$ are nicely correlated signals in these two limits.

Fig. 8 shows the damping of the condensate fraction $f_{0}$ for increasing $U / J$ and fixed force. The behavior is very similar to the momentum distribution, but easier to fit to extract a typical damping time $\tau$. We extract the typical damping time from the normalized condensate fraction time-evolution by fitting the curves using the function $f(t)=a+(1-a) /\left(1+(t / \tau)^{b}\right)$. $a$ is the asymptotic value of the fonction, and $\tau$ is such that the curve has decayed of half its distance from 1 to $a$. The exponent $b$ is found to vary between 1 and 5 in the fits but its value does not significantly change $\tau$ which is the quantity of inter- est. Good fits are obtained at small $U$ while oscillations spoil the fit at large $U$, which nevertheless provides a reasonable estimate of the damping time. The main plot and the inset supports that the $\tau \mathrm{s}$ become much longer near the two integrable points.

\section{Thermalization}

In addition to the short-time behavior, the difference between integrable and non-integrable regimes can show up in the long-time average value $\bar{O}$. We see that both the features of the diagonal distribution $p_{n}$ and the behavior of the observables with energy intervene in Eq. (12). If one wants to study thermodynamics only, the energy distribution $p_{n}$ is the only useful quantity. In the case of a generic distribution, the equivalence of ensemble should be sufficient to provide the same thermodynamics as usual thermal ensembles [25]. If one wants to compare time-averaged observables $\bar{O}$ to those obtained from thermal ensembles, the hypothesis that $O_{n n} \sim O(E)$ hardly varies within the energy shell given by the typical energy fluctuations naturally leads to the identification with $\bar{O}$ and, therefore, to thermalized observables in this sense. Justification of this idea (sometimes dubbed as the Eigenstate thermalization hypothesis, or ETH) has been proposed from quantum chaos arguments [26, 27] and studied numerically [28, 29]. Some extensions and more details prescription of this statement have been discussed more recently [30], stressing the importance of the correlations between $p_{n}$ and $O_{n n}$. In this context, integrability or the proximity to integrable points on finite systems has been shown to lead, most of the time, to nonthermalized observables [17, 28, 30, 35]. The particular situation of an interaction quench in the Bose-Hubbard model which possesses integrable limits, has been discussed in depth in Refs. 30, 32, 36, and 37. Of course, the above approaches suffer from limitations in their applicability. For instance it is expected to work best at high energies where quantum chaos describes well the states. Yet, this corresponds to hightemperature where the physics is in general not so interesting. Another issue comes when taking the thermodynamic limit $N \rightarrow \infty$ after or before time-averaging.

We now turn to the calculation of the time-averaged expectation $\bar{O}$ of an observable compared to its thermal prediction $O^{\text {th }} . \bar{O}$ is directly obtained from (12) from full diagonalization of the Hamiltonian on small systems (we use $L=12$ and $N=6$ ) and from computing the quench distribution $p_{n}$ exactly. $O^{\text {th }}$ is obtained using calculation in the canonical ensemble. A microcanonical description is also possible but it is in practice less transparent as one has to tune to energy window. Usually, the outcome does not depend much on the choice of the distribution at sufficiently high energies (temperatures) and looking at simple observables; Besides, the equivalence of ensemble suggests that both ensemble should in principle yield the same results in the thermodynamical limit, i.e. for large enough systems. The temperature $T_{B}$ of the Boltzmann distribution is directly obtained by demanding that the mean-energy should be the same as the mean-energy 

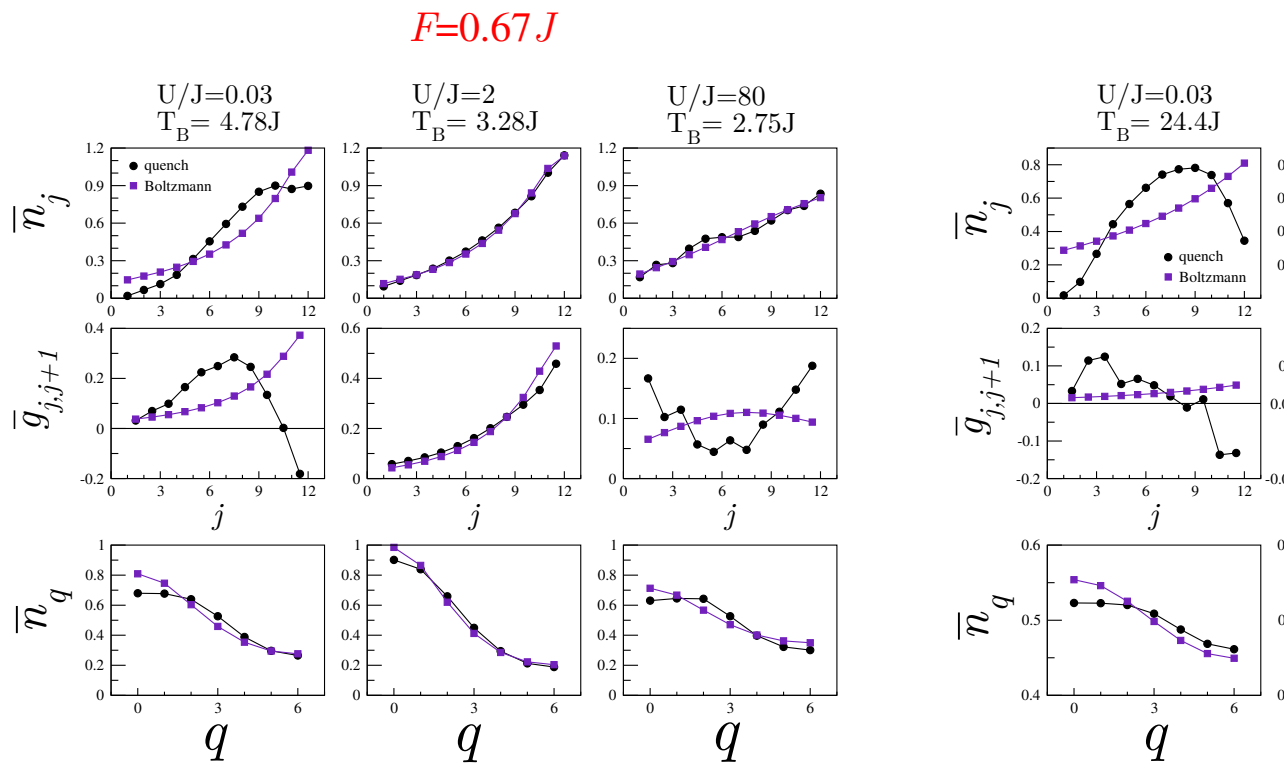

$$
F=1.67 J
$$
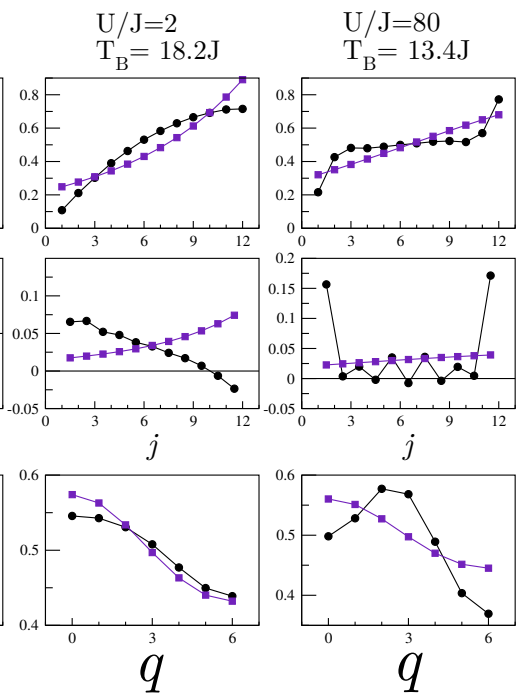

FIG. 9. (color online). Comparison between time-averaged profiles obtained after the quench and thermalized profiles with the same meanenergy (in a closed box). Left: for a force $F$ large enough to put enough energy in the system, but not too large to destroy non-integrability, the gas looks thermalized in the non-integrable regime $U / J=2.0$, while clear deviations from thermalization are observed close to the integrable limits $U=0, \infty$. Right: When the force is larger, this finite-size system $(L=12)$ looks too integrable to observe approximately thermalized behaviors. In both cases, $T_{B}$ indicates the temperature obtained from the mean-energy using the canonical ensemble.

of the system after the quench $\langle E\rangle=\sum_{n} p_{n} E_{n}$. Then, the Boltzmann weights read $p_{n}=e^{-E_{n} / k_{B} T} / Z$ with $Z$ the partition function and we plug them in (12) to get $O^{\text {th }}$.

The goal is now to see whether deviations from thermalization are related to the integrability of the model studied previously. In Fig. 9, we show the profiles for the three simplest observables for quenches with moderate forces $F$ and three typical values of $U / J$ respectively close to the non-interacting limit, deep in the non-integrable region and close to the HCB limit. One must not choose a force which is too small, otherwise little energy is put in the system, the temperature would be too small and the discussion possibly spoiled by finite-size effects [32]. If the force is too large, we know from Fig. 12 that the system looks integrable for all $U / J$. We find that $F / J \simeq 0.5,1$ is a reasonable compromise to use the quench protocol to probe the relation between thermalization and integrability in this setup. Indeed, in Fig. 9, we observe that for $F / J=0.67$, all profiles agree well with the thermal predictions for $U / J=2$, deep in the non-integrable regime. Close to the integrable points, deviations are found but the effect depends on the chosen observable. We find that the local kinetic energy $g_{j, j+1}$ is the most suitable observable to probe the effect of the integrability (something also discussed in Ref. 33). For $F / J=1.67$, it is much harder to reach thermalization, probably due to the near integrable features of the Hamiltonian for this small system.

In order to quantify the deviation from the thermal prediction, we average over the sites $j$ (or momentum $q$ for $n_{q}$ ) the

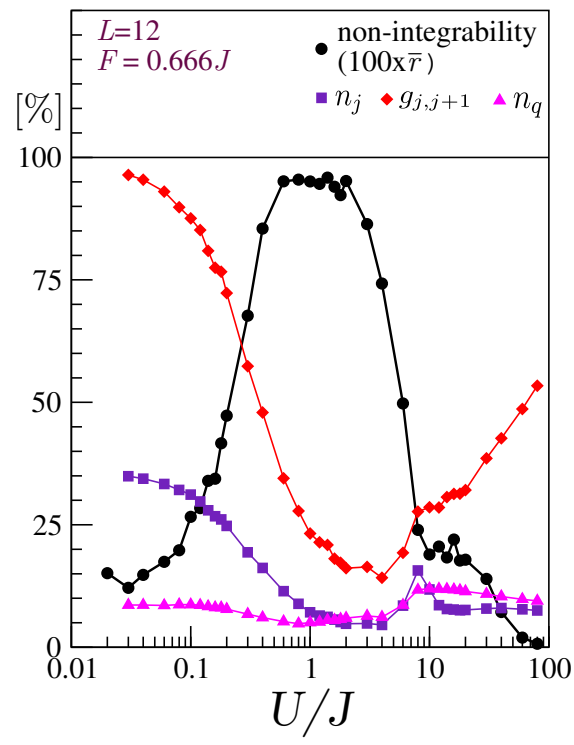

FIG. 10. (color online). Relative distance (in \%) between the timeaverage and the thermal expectations of an observable v.s. $U / J$ (density $n_{j}$, kinetic energy $g_{j, j+1}$, momentum distribution $\left.n_{q}\right) . \bar{r}$ measures the non-integrability of the model.

relative distance from thermal prediction and dub it:

$$
\delta O=\frac{1}{L} \sum_{j}^{L}\left|\frac{\bar{O}_{j}-O_{j}^{\mathrm{th}}}{O_{j}^{\mathrm{th}}}\right| .
$$

This is the quantity plotted in percentage in Fig. 10 as a function of $U / J$. We qualitatively expect that non-thermalized 
regimes occur close to integrable points [28, 31--33]. This is well observed in Fig. 10 for which the integrability measure, denoted by $\bar{r}$ (see Appendix B for definitions), is manifestly correlated to the non-thermalization measure. The advantage of this setup with respect to interaction quenches [30, 32, 36, 37] is that the ratio $U / J$ is kept fixed. In other words, it decouples the quenching parameter from the parameter which mainly controls integrability.

\section{CONCLUSION}

We introduced and characterized a remarkable setup, the "quantum Galileo ramp", which can be used to create entangled many-body wave-packets. We further provide simple views on how to manipulate the condensate afterwards. An important result is that the asymptotic non-equilibrium entanglement entropy is quantitatively related to the equilibrium initial one in the $\mathrm{HCB}$ limit. In addition, non-trivial Josephson-like oscillations are found and we show the setup is well suited to study the role of integrability on damping and thermalization. We underline that results on HCB concerning the local density are also valid for free fermions. Beyond the field of cold atoms, the setup could be developed for polariton condensates [38].

\section{Acknowledgements}

This work is supported by ANR-09-BLAN-0098-01. M. C. benefited from the International Graduate College on Statistical Physics and Complex Systems between the universities of Nancy and Leipzig.

\section{Appendix A: Neglecting the tunneling toward the upper Bloch band}

Experimentally, tunneling to upper Bloch band could occur if the band is to close in energy to the states of the trapped particles. Indeed, under the constant force $F$, Bloch oscillations may be ruled out by Landau-Zener interband transitions (and particles may possibly escape into the continuum if the upper gaps are too small). The rate at which this escape toward the upper band is expected is roughly given by the Landau-Zener tunneling formula $e^{-c \delta^{2} / F}$ [5, 39] where $\delta$ is the interband energy gap and $c$ is a constant depending in particular on the recoil energy ( $E_{R}=\Delta$ here). To avoid the escape of particles toward the upper band one needs accordingly to apply a sufficiently low force such that $F \ll c \delta^{2}$. On the other hand, one needs a sufficiently high force in order to scan the first Brillouin zone within a reasonable small time (from an experimental point of view) [39].
Appendix B: Spectral features and chaos in the box geometry (without a propagative band)

\section{Hamiltonian}

We study using Lanczos and full diagonalization techniques the Bose-Hubbard Hamiltonian in box of size $L$ and subjected to a linear potential $V_{j}=V_{A}-F j$, where $F$ is the slope (or force):

$\mathcal{H}=-J \sum_{j}\left[b_{j+1}^{\dagger} b_{j}+\right.$ h.c. $]+\frac{U}{2} \sum_{j} n_{j}\left(n_{j}-1\right)+\sum_{j} V_{j} n_{j}$

with $V_{j}$ the external potential. If the onsite boson cutoff $M=$ 1 , we have the integrable XX model while if $M=N$ we recover the Bose-Hubbard model. In the following, all data are for a density $\rho=N / L=1 / 2$ (superfluid regime) and full diagonalization are done with $L=12$ (Hilbert space size of about 12000 states), and Lanczos calculations up to $L=18$.

This model has been investigated in a similar context, analyzing the effect of interactions on BO and its regular/chaotics regimes. The main known results are that a new period, in addition to $\tau_{B}$, emerges in the BO for finite $U$ and in the strong force limit, which reads $2 \pi / U$ [9]. Chaotic motion is found for small $F$ and in the presence of interactions, leading to a damping of the BO [24]. A strong reduction of damping has been observed experimentally [10] when $U \rightarrow 0$. The Mott regime $U \gg J$ of the $\mathrm{BO}$ has also been briefly investigated in Ref. 40. Level statistics and the chaotic nature of the spectrum has been partially analyzed in Ref. 24 and 41 . Approximate methods such as discrete non-linear Schrödinger equation and mean-field theory have been also used to study the dynamics of this model [40, 42, 43]. We lastly mention that the hard-core boson dynamics in a tilted bichromatic lattice (which possesses a gap in the single-particle dispersion relation) has been investigated in Ref. 44

\section{Density of states}

We first discuss the nature of the many-body density of states (DOS) of the Hamiltonian as a function of the two parameters $U / J$ and $F / J$. There are three energy scales in the problem : the hopping $J$ which sets the bandwith of the single-particle energies, the local interaction $U$ and the external potential $\operatorname{ramp} F$. These typical energies are visible in the many-body DOS of the spectrum as seen in Fig. 11. When only $J$ is present, the DOS has a gaussian shape centered around zero energy. In the large- $F$ limit, we observe equally-spaced peaks separated by the energy $F$. This structure is reminiscent of the single-particle Wannier-Stark ladder spectrum. It is as well trivially understood at the many-body level when $F \gg J$ since $F$ corresponds to the potential energy cost when a particle jumps from one site to its neighbor. Similarly, when $F=0$ and $U \gg J$, elementary processes corresponding to changing the onsite number of particles yield an equally-spaced spectrum of energy $U$ (Mott lobes). Notice that $F$ rapidly kills these lobes as we see on Fig. 11 that 

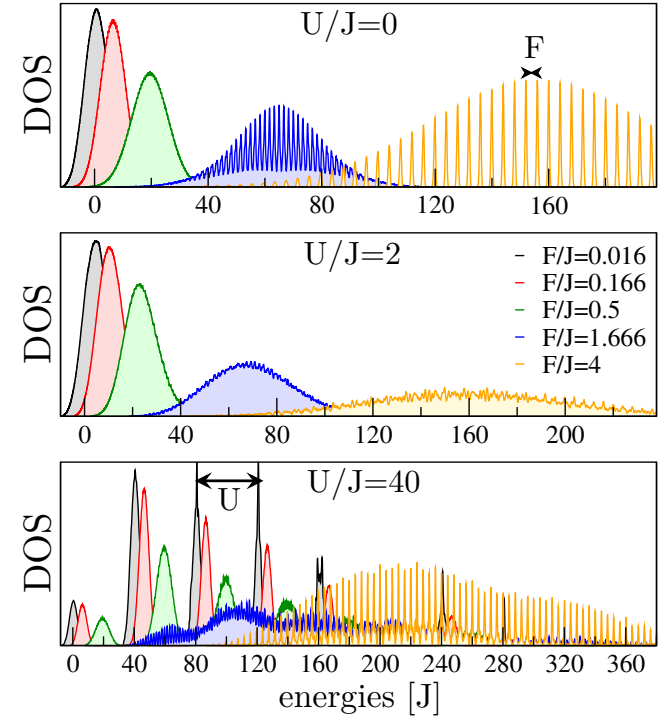

FIG. 11. (color online). Evolution of the density of states of the many-body Hamiltonian with slope $F$ for different $U$. We observe main bands separated by $U$ at large $U$ or by $F$ at large $F$.

$F / J \gtrsim 1$ is sufficient to destroy the lobes in the DOS.

\section{Signatures of integrability and chaoticity in the spectrum}

In order to study the non-integrable nature of the Hamiltonian (B1) which governs the time-evolution, we use level and wave-function statistics (not shown). However, we see that the usual unfolding procedure for the spectrum will be plagued in the large $F$ or large $U$ regimes, due to the peak structure of the DOS. Following Refs. 45$]$ and 46 , we use the statistics of the ratio of consecutive level spacings $r_{n}=$ $\min \left(\delta_{n}, \delta_{n-1}\right) / \max \left(\delta_{n}, \delta_{n-1}\right)$ where $\delta_{n}=E_{n+1}-E_{n}$ is the level spacing and $E_{n}$ are the energies. Probability distributions $P(r)$ are then compared to poissonian and gaussian orthogonal ensemble (GOE) statistics.

An example for the model under study is given in Fig. 12 in which we observe both regular (Poisson) and chaotic (GOE) distributions, depending on the parameters. In order to extract a simple number to be plotted against the parameters, we use the normalized mean ratio defined by $\bar{r}=$ $\left(\langle r\rangle-\langle r\rangle_{\text {Poisson }}\right) /\left(\langle r\rangle_{\mathrm{GOE}}-\langle r\rangle_{\text {Poisson }}\right)$, which is 1 in the nonintegrable regime and 0 in the integrable regime. When $F \neq$ 0 , the reflection symmetry of the box is lost and the Hamiltonian (B1) has no spatial symmetries which could plague the level statistics if not taken into account. As discussed in Ref. 45 in the $F=0$ case, only the $U=0$ and $J=0$ limits are integrable, Bethe-ansatz predicting the Hamiltonian is non-integrable as soon as $U, J \neq 0$. Still, because of finite size effects, small and large $U / J$ regions look almost integrable on a finite cluster. The thermodynamic limit is particularly hard to investigate numerically, although the trend is compatible with the Bethe-ansatz predicting the Hamiltonian is non-integrable as soon as $U, J \neq 0$. Still, because of

(a)
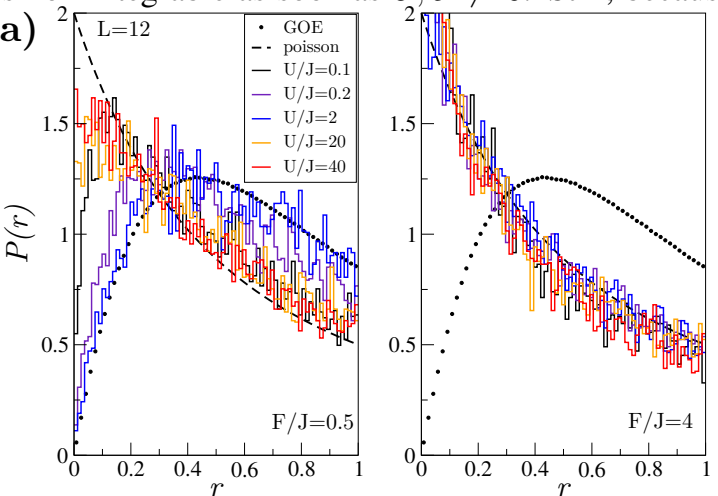

(b)

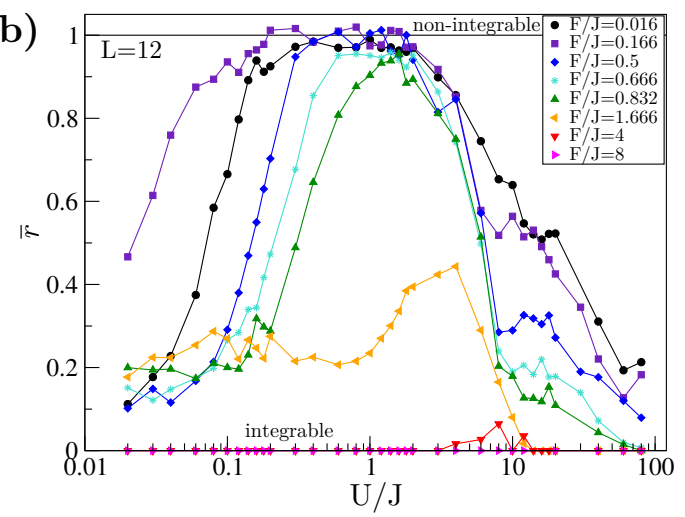

FIG. 12. (color online). (a) Probability distributions of the mean adjacent level spacing ratio $P(r)$ as a function of $U / J$ for two different slopes $F$. (b) Evolution of the normalized mean adjacent level spacing ratio $\langle r\rangle$ as a function of $U / J$ for different slope $F$.

finite size effects, small and large $U / J$ regions look almost integrable on a finite cluster. The thermodynamic limit is particularly hard to investigate numerically, although the trend is compatible with the Bethe-ansatz perspective [45]. We then expect [24, 41] the large $U$ and large $F$ regime to be close to the integrable classical limit $(J=0)$, at least on a finite chain. The results for different $F$ as a function of $U / J$ are displayed on Fig. 12 and we see that for $F=4 J$, the distribution looks always poissonian. In order to observe non-integrable effects, we must not choose $F$ too large $(F \lesssim 1$ for this $\rho=1 / 2$ and $L=12$ ). We also notice from this figure that the optimal range of $U$ to be in the non-integrable regime depends on $F$.
[1] I. Bloch, J. Dalibard, and W. Zwerger, Rev. Mod. Phys. 80, 885 (2008).

[2] J. Dziarmaga, Advances in Physics 59, 1063 (2010);
A. Polkovnikov, K. Sengupta, A. Silva, and M. Vengalattore, Rev. Mod. Phys. 83, 863 (2011).

[3] F. Bloch, Z. Phys 52, 555 (1928); C. Zener, Proc. R. Soc. Lon- 
don Ser. A 145, 523 (1934).

[4] C. Waschke et al., Phys. Rev. Lett. 70, 3319 (1993).

[5] M. Ben Dahan et al., Phys. Rev. Lett. 76, 4508 (1996).

[6] V. Agarwal et al., Phys. Rev. Lett. 92, 097401 (2004).

[7] O. Morsch et al., Phys. Rev. Lett. 87, 140402 (2001).

[8] Z. He et al., Phys. Rev. E 76, 056605 (2007).

[9] A. R. Kolovsky, Phys. Rev. Lett. 90, 213002 (2003).

[10] M. Gustavsson et al., Phys. Rev. Lett. 100, 080404 (2008).

[11] F. Heidrich-Meisner et al., Phys. Rev. A 80, 041603 (2009).

[12] J. Billy et al., Nature 453, 891 (2008); G. Roati et al., Nature 453, 895 (2008).

[13] I. Bloch et al., Phys. Rev. Lett. 87, 030401 (2001).

[14] B. T. Seaman et al., Phys. Rev. A 75, 023615 (2007).

[15] M. Rigol and A. Muramatsu, Phys. Rev. Lett. 93, 230404 (2004).

[16] D. Karevski, Eur. Phys. J. B 27, 147 (2002); G. M. Schuetz and S. Trimper, Europhys. Lett. 47, 164 (1999); S. Dorosz, T. Platini, and D. Karevski, Phys. Rev. E 77, 051120 (2008); T. Platini and D. Karevski, J. Phys. A 40, 1711 (2007); D. Karevski and T. Platini, Phys. Rev. Lett. 102, 207207 (2009); T. Platini, R. J. Harris and D. Karevski, J. Phys. A 43, 135003 (2010); Y. Ogata, Phys. Rev. E 66, 016135 (2002); Y. Ogata, Phys. Rev. E 66, 066123 (2002); W. H. Aschbacher and C.-A. Pillet, J. Stat. Phys. 112, 1153 (2003).

[17] M. Rigol, A. Muramatsu, and M. Olshanii, Phys. Rev. A 74, 053616 (2006); M. Rigol, V. Dunjko, V. Yurovsky, and M. Olshanii, Phys. Rev. Lett. 98, 050405 (2007).

[18] S. R. White, Phys. Rev. Lett. 69, 2863 (1992); S. R. White, Phys. Rev. B 48, 10345 (1993); U. Schollwöck, Rev. Mod. Phys. 77, 259 (2005).

[19] G. Vidal, Phys. Rev. Lett. 91, 147902 (2003); Phys. Rev. Lett. 93, 040502 (2004).

[20] S. R. White and A. E. Feiguin, Phys. Rev. Lett. 93, 076401 (2004);

[21] A. J. Daley, C. Kollath, U. Schollwöck, and G. Vidal, J. Stat. Mech. P04005 (2004).

[22] U. Schollwöck and S. R. White, AIP Conf. Proc. 816, 155
(2006); G. D. Chiara, M. Rizzi, D. Rossini, and S. Montangero, J. Comput. Theor. Nanosci. 5, 1277 (2008).

[23] P. Calabrese, M. Mintchev, and E. Vicari, Phys. Rev. Lett. 107, 020601 (2011); Europhys. Lett. 97, 20009 (2012);

[24] A. Buchleitner and A. R. Kolovsky, Phys. Rev. Lett. 91, 253002 (2003).

[25] G. Roux, Phys. Rev. A 82, 037602 (2010).

[26] A. Peres, Phys. Rev. A 30, 1610 (1984); Phys. Rev. A 30, 504 (1984).

[27] J. M. Deutsch, Phys. Rev. A 43, 2046 (1991); M. Srednicki, Phys. Rev. E 50, 888 (1994).

[28] M. Rigol, V. Dunjko, and M. Olshanii, Nature 452, 854 (2008).

[29] M. Feingold, N. Moiseyev, and A. Peres, Phys. Rev. A 30, 509 (1984); R. V. Jensen and R. Shankar, Phys. Rev. Lett. 54, 1879 (1985); M. Feingold and A. Peres, Phys. Rev. A 34, 591 (1986).

[30] G. Biroli, C. Kollath, and A. M. Läuchli, Phys. Rev. Lett. 105, 250401 (2010).

[31] M. Rigol, Phys. Rev. Lett. 103, 100403 (2009).

[32] G. Roux, Phys. Rev. A 81, 053604 (2010).

[33] L. F. Santos and M. Rigol, Phys. Rev. E 81, 036206 (2010).

[34] L. F. Santos and M. Rigol, Phys. Rev. E 82, 031130 (2010).

[35] A. Iucci and M. A. Cazalilla, Phys. Rev. A 80, 063619 (2009); New Journal of Physics 12, 055019 (2010).

[36] C. Kollath, A. M. Läuchli, and E. Altman, Phys. Rev. Lett. 98, 180601 (2007).

[37] G. Roux, Phys. Rev. A 79, 021608(R) (2009).

[38] J. Kasprzak et al., Nature 443, 409 (2006).

[39] E. Peik et al., Phys. Rev. A 55, 2989 (1997).

[40] A. R. Kolovsky, Phys. Rev. A 70, 015604 (2004).

[41] A. R. Kolovsky and A. Buchleitner, Phys. Rev. E 68, 056213 (2003).

[42] A. R. Kolovsky, H. J. Korsch, and E.-M. Graefe, Phys. Rev. A 80, 023617 (2009).

[43] A. R. Kolovsky, Phys. Rev. A 82, 011601 (2010).

[44] X. Cai, S. Chen, and Y. Wang, Phys. Rev. A 84, 033605 (2011).

[45] C. Kollath, G. Roux, G. Biroli, and A. M. Läuchli, J. Stat. Mech. P08011 (2010).

[46] V. Oganesyan and D. A. Huse, Phys. Rev. B 75, 155111 (2007). 